m06.p12

\section{Structural Insight into Intermediate Filament Assembly Using Small-Angle X-ray Scattering}

A.V. Sokolova ${ }^{a}$, L. Kreplak ${ }^{\text {, }}$ L.P. Kurochkina ${ }^{a}$, D.I. Svergun ${ }^{\mathrm{c}}$, T. Wedig ${ }^{\mathrm{d}}$, H. Herrmann ${ }^{\mathrm{d}}$, U. Aebi ${ }^{\mathrm{b}}$, and $\underline{\text { S.V. Strelkov }}^{\mathrm{e}}$

${ }^{a}$ Institute of Bioorganic Chemistry, Moscow, Russia, ${ }^{b}$ M.E. Mueller Institute, Biozentrum Basel, Switzerland, ${ }^{c}$ EMBL, Hamburg, Germany, ${ }^{d}$ German Cancer Research Centre, Heidelberg, Germany, ${ }^{e}$ Department of Pharmaceutical Sciences, Catholic University of Leuven, Belgium. E-mail: sergei.strelkov@pharm.kuleuven.be

Keywords: intermediate filaments, molecular architecture self-assembly, small angle X-ray scattering

$10 \mathrm{~nm}$ wide intermediate filaments (IFs), along with microtubules and actin filaments, constitute the cytoskeleton of metazoan cells. While crystallographic data on the dimer representing the elementary IF 'building block' have recently become available, little structural detail is known on both the mature IF architecture and their assembly pathway. Here we have applied solution small-angle X-ray scattering (SAXS) to investigate the in vitro assembly of a $53 \mathrm{kDa}$ human IF protein vimentin by systematically varying the $\mathrm{pH}$ and ionic strength conditions, and complemented these experiments by electron microscopy and analytical ultracentrifugation. While vimentin solution in $5 \mathrm{mM}$ Tris- $\mathrm{HCl}(\mathrm{pH} 8.4)$ contains predominantly tetramers, further lateral assembly induced by addition of 20 $\mathrm{mM} \mathrm{NaCl}$ leads to the formation of a distinct octameric intermediate. Four octamers eventually associate into the unitlength filaments (ULFs) that anneal longitudinally. Based on the SAXS experiments supplemented by crystallographic data and additional structural constraints, first ever three-dimensional molecular models of vimentin tetramer, octamer and the ULF are constructed. Within each of the three oligomers, the adjacent dimers are aligned exclusively in a half-staggered antiparallel mode $\left(\mathrm{A}_{11}\right)$ with a distance of 3.2-3.4 nm between their axes. While a dynamic structure, the ULF appears to have average dimensions of $9 \mathrm{~nm} \times 16 \mathrm{~nm} \times 65 \mathrm{~nm}$ and a roughly even mass distribution over the cross-section. Together with most recent crystal structures of vimentin fragments, these results represent a major advance towards unveiling the structural basis of both the normal IF function and the mechanism of human disease caused by mutations in IF proteins.

\section{m06.p13}

\section{Structure of the Insulin Receptor Ectodomain Homodimer}

Neil McKern, Michael Lawrence, Victor Streltsov*, Meizhen Lou, Timothy Adams, George Lovrecz, John Bentley, Peter Hoyne, Maurice Frenkel, Colin Ward

CSIRO Molecular and Health Technologies, Melbourne, Victoria, Australia. E-mail:victor.strelstov@csiro.au

\section{Keywords: insulin receptor, X-ray structure, IR} ectodomain

The insulin receptor (IR) is a phylogenetically ancient tyrosine kinase receptor found in organisms as primitive as cnidarians and insects. The key role of the insulin receptor (IR) is in glucose uptake and metabolism by muscle and fat. Dysfunctional IR signaling has been implicated in diseases including type I and type II diabetes, dementia and cancer. The IR exists as two splice variant isoforms IR-A and IR-B. The IR-B isoform is responsible for signaling metabolic responses. In contrast, IR-A signals predominantly mitogenic responses, is the preferentially expressed isoform in several cancers and is capable of binding insulin-like growth factor receptor (IGF-II) with high affinity. Here we present the crystal structure of the IR-A ectodomain dimer. The structure reveals, for the first time, the domain arrangement in the disulphide-linked ectodomain dimer and shows that it adopts a folded-over conformation that places the ligand-binding regions in juxtaposition. 